Sensors 2008, 8, 1819-1831

sensors

ISSN 1424-8220

(C) 2008 by MDPI

www.mdpi.org/sensors

Full Research Paper

\title{
A New PC and LabVIEW Package Based System for Electrochemical Investigations
}

\section{Zoran Stević ${ }^{*}$, Zoran Andjelković and Dejan Antić}

University of Belgrade, Technical Faculty in Bor, VJ 12, 19210 Bor, Serbia; E-mails: Z. Andjelković: jaz@eunet.yu; D. Antić: dantic@tf.bor.ac.yu

* Author to whom correspondence should be addressed; E-mail: zstevic@tf.bor.ac.yu

Received: 28 February 2008 / Accepted: 12 March 2008 / Published: 15 March 2008

\begin{abstract}
The paper describes a new PC and LabVIEW software package based system for electrochemical research. An overview of well known electrochemical methods, such as potential measurements, galvanostatic and potentiostatic method, cyclic voltammetry and EIS is given. Electrochemical impedance spectroscopy has been adapted for systems containing large capacitances. For signal generation and recording of the response of investigated electrochemical cell, a measurement and control system was developed, based on a PC P4. The rest of the hardware consists of a commercially available AD-DA converter and an external interface for analog signal processing. The interface is a result of authors own research. The software platform for desired measurement methods is LabVIEW 8.2 package, which is regarded as a high standard in the area of modern virtual instruments. The developed system was adjusted, tested and compared with commercially available system and ORCAD simulation.
\end{abstract}

Keywords: Electrochemical Measurements, Measurement System, Electrochemical Impedance Spectroscopy, Cyclic Voltammetry

\section{Introduction}

Electrochemical investigation methods are widely used for characterization of different kinds of materials, as well as of the processes in systems where the electrochemical reactions take part [1, 2]. There is a series of well known methods, but some new methods from the electrotechnical area have been introduced. So, first of all an overview of the standard electrochemical methods and parameters is 
given, beginning with potential measurement and simple methods such as chronopotentiometry and chronoamperometry, up to electrochemical impedance spectroscopy. The last named method is adapted for systems containing large capacitances that became current with the appearance of electrochemical super capacitors. New methods are Dirack voltage excitation and Dirack current excitation. The measurement system described here is a new, updated version of one previously developed by the same authors [3] that allows application of the following electrochemical methods: measurement of open circuit potentials, chronopotentiometry, chronoamperometry, galvanostatic and potentiostatic methods, Dirack voltage excitation, galvanodynamic methods, cyclic voltammetry and electrochemical impedance spectroscopy. The system is suitable for electrochemical laboratories at faculties and institutes where it could replace expensive and/or old measurement equipment, increasing work comfort and the quality of obtained results.

\section{Hardware}

For signal generation and data acquisition a measuring and control system based on a Pentium 4 PC was developed. Besides the PC, the hardware consists of an ADDA converter and an external interface for analog signal conditioning [4-7]. ADDA conversion is performed using a commercially available NI 6251converter (National Instruments). National Instruments $\mathrm{M}$ series high-speed multifunction data acquisition (DAQ) devices are optimized for superior accuracy at fast sampling rates. They have an onboard NI-PGIA2 amplifier designed for fast settling times and high scanning rates, ensuring 16-bit accuracy even when measuring all channels at maximum speeds. All high speed devices have a minimum of 16 analog inputs, 24 digital I/O lines, seven programmable input ranges, analog and digital triggering, and two counter/timers [8].

Measurement interface designed for the needs of the electrochemical investigations by controlled current or voltage excitation has the following characteristics:

- two control voltage inputs $\pm 10 \mathrm{~V}$,

- one measuring current input $\pm 100 \mathrm{~mA}$.

- $\quad$ one voltage output $\pm 10 \mathrm{~V}$ for input current of $\pm 100 \mathrm{~mA}$,

- one voltage input for the reference potential recording,

- the reference electrode input resistance higher then $10^{12} \Omega$,

- one the three-electrode output for electrochemical cell with the next possibilities:

- voltage range $\pm 5 \mathrm{~V}$ with the possibility of superimposing the small signal in the range of $\pm 10 \mathrm{mV}$,

- current range $\pm 100 \mathrm{~mA}$.

Characteristics mentioned above have been realized by the block scheme given in Figure 1.

Considering that gain of amplifier $\mathrm{A} 1$ is preset to $\mathrm{A} 1=1$, voltage on analog input channel zero of the analog to digital converter (A/D) is going to be:

$$
V_{\text {AICH } 0}=A_{1} \cdot V_{R E}=V_{R E}=-V_{W E-R E}
$$

where: VRE - is the voltage of the reference electrode relative to the device ground; VWE-RE - is the potential difference (voltage) between the working and reference electrodes. 
Figure 1. The block diagram of the interface.

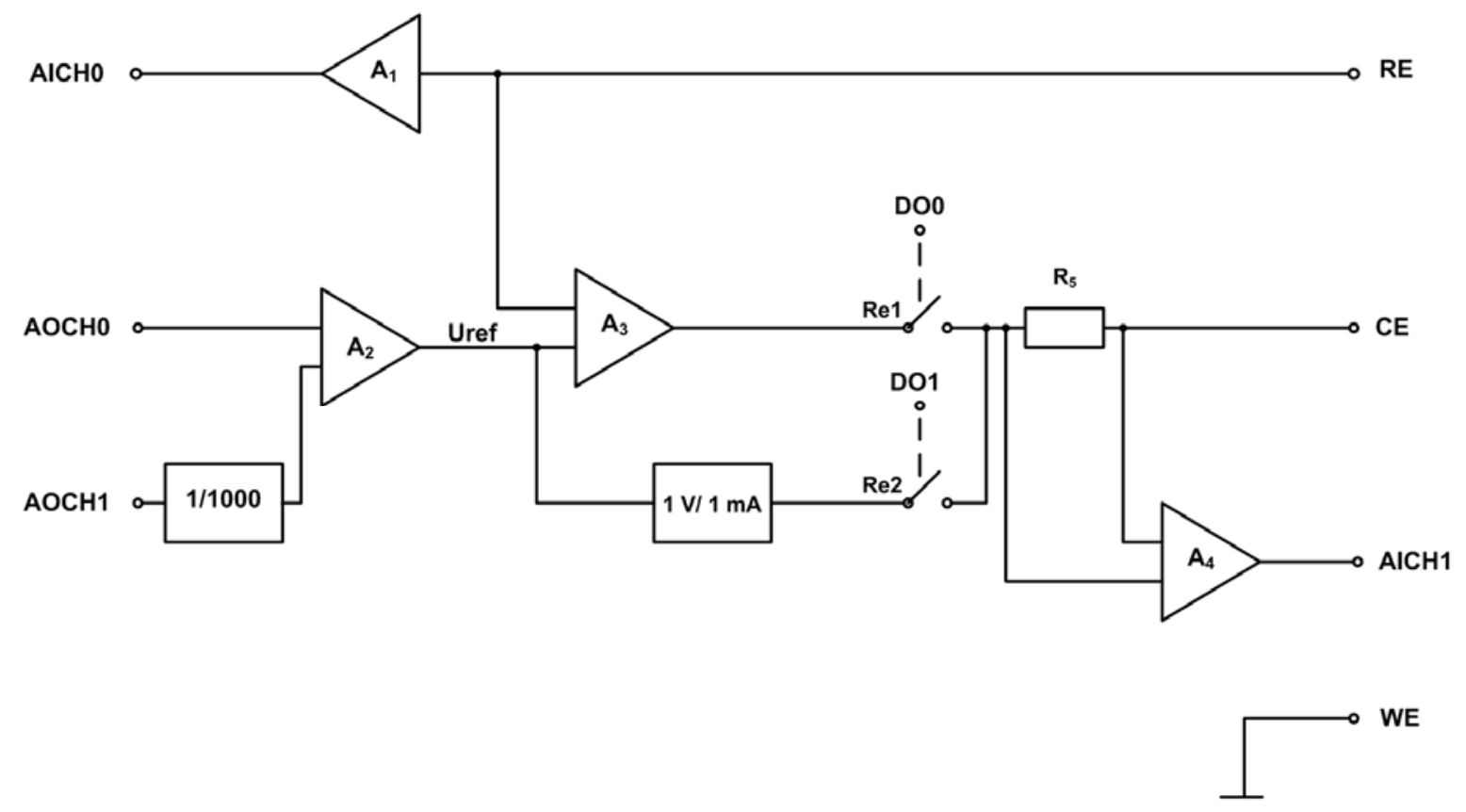

The given shape of the voltage signal is being brought from the analog output - the channel (AOCH0) upon the amplifier A2 and then it is added to some other controlling voltage (AOCH1) weakened by 1000 times. If the gain of the collector $A 2$ is set to one (A2=1), output voltage of the system is going to be given by:

$$
V_{W E}=-V_{R E}=-V_{A O C H 0}-\frac{V_{A O C H 1}}{1000}
$$

where: VRE - is the voltage of the reference electrode with respect to the device ground; VAOCH0 is the voltage on analog output channel zero of digital to analog converter (D/A); VAOCH1 - is the voltage on analog output channel one of $\mathrm{D} / \mathrm{A}$ converter.

It is possible to achieve the output of $\pm 10 \mathrm{~V}$ accompanied by the superimposed $\pm 10 \mathrm{mV}$ (for controlling voltages $\pm 10 \mathrm{~V}$ ). The output amplifier - the comparator $A_{3}$ reaches the same voltage upon its own output in relation to the reference electrode (RE). The working electrode (WE) has been grounded and it is the reason why the counter electrode (CE) is negatively polarized in case it is required to obtain the positive polarization of the working electrode versus the counter electrode.

The electrochemical cell is protected from uncontrolled voltages or currents, during the process of initialization of hardware, in that way that the two relays Re1 and Re2 are being switched off until the regular start of the chosen method.

In case of a current regime, a converter (U/I) whose relation of $10 \mathrm{~mA} / 1 \mathrm{~V}$ provides the output current $\pm 100 \mathrm{~mA}$ for the controlling voltage of $\pm 10 \mathrm{~V}$ has been anticipated. The regime choice is done by a software, switching off the relays Re1 or Re2 through the digital outputs DO0 or DO1. Subassemblies of the described block scheme have been done in accordance with the standards, paying a special attention to the input resistance of the reference electrode (RE).

Considering that accepted values of resistor R5=1 $\Omega$ and gain of amplifier A4=100, voltage on analog input channel one (AICH1) of the A/D converter is going to be: 


$$
V_{\mathrm{AICH} 1}=I_{C E} \cdot R_{5} \cdot A_{4}=100 \cdot I_{C E}=-100 \cdot I_{W E}
$$

where: ICE - is current on counter electrode; IWE - is current on working electrode; R5 - is resistance.

The output current is being measured in such a way that the voltage drop is monitored by means of the differential amplifier $\left(A_{4}\right)$ on the resistor $R_{5}$. The output of the amplifier $A_{4}$ is being led upon the analog input AICHI1. Analog input voltage on channel one (AICH1) of A/D converter is going to be $\pm 10 \mathrm{~V}$ for working current scale of $\pm 100 \mathrm{~mA}$.

\section{Software}

The software platform for predicted measurement methods was National Instruments LabVIEW 8.2 package, which is regarded as a high standard in the area of modern virtual instruments [9-11]. LabVIEW is based on the principles of virtual instruments with the graphical user interface. Graphical user interface has two windows:

- Front Panel for process control and monitoring,

- Application diagram (Block Diagram) which presents used virtual instruments, relations between them, the course of signals and error detection.

In LabVIEW, one builds a user interface by using a set of tools and objects. The user interface is known as the front panel. One then add code using graphical representations of functions to control the front panel objects. The block diagram contains this code.

As an example, the Block Diagrams as well as Front Panels has been showed for the characteristic electrochemical methods.

\subsection{Measuring of Open Circuit Potential}

For a potential measurement of the working electrode, that is, the voltage in relation to the reference electrode, there has been installed the application with one input channel (AICH0).

The reading of the incoming signal is done by the block DAQmx Read, defining the following: the physical channel where the reading is being done, the way of connecting the channels on the measuring system, the measuring range of the signal, units by which the reading is done, as well as the number and type of measurements. Many a time read input voltage, in this case 1000 times, is being led in the block for averaging - Mean. The average value of the measured potential is being showed on the digital display of the instrument as well as in the form of a graph $E=f(t)$ by the block Waveform Chart. The described cycle of reading and showing the potential has been repeating until the user does not terminate the execution by pressing the STOP button.

The measured values of the potentials are transformed into a two-dimensional array, transferred and recorded in the text file, whose name and path are determined by the user. In the figures 2 and 3 , there have been showed the front panel and block diagram of the instrument for measuring open circuit potential with controls for choosing the measuring range and the file name, as well as indicators (current time $t[s]$, the measured potential $E[V]$ ) and the graph $E=f(t)$. 
Figure 2. Front Panel of instrument for measuring the open circuit potential.

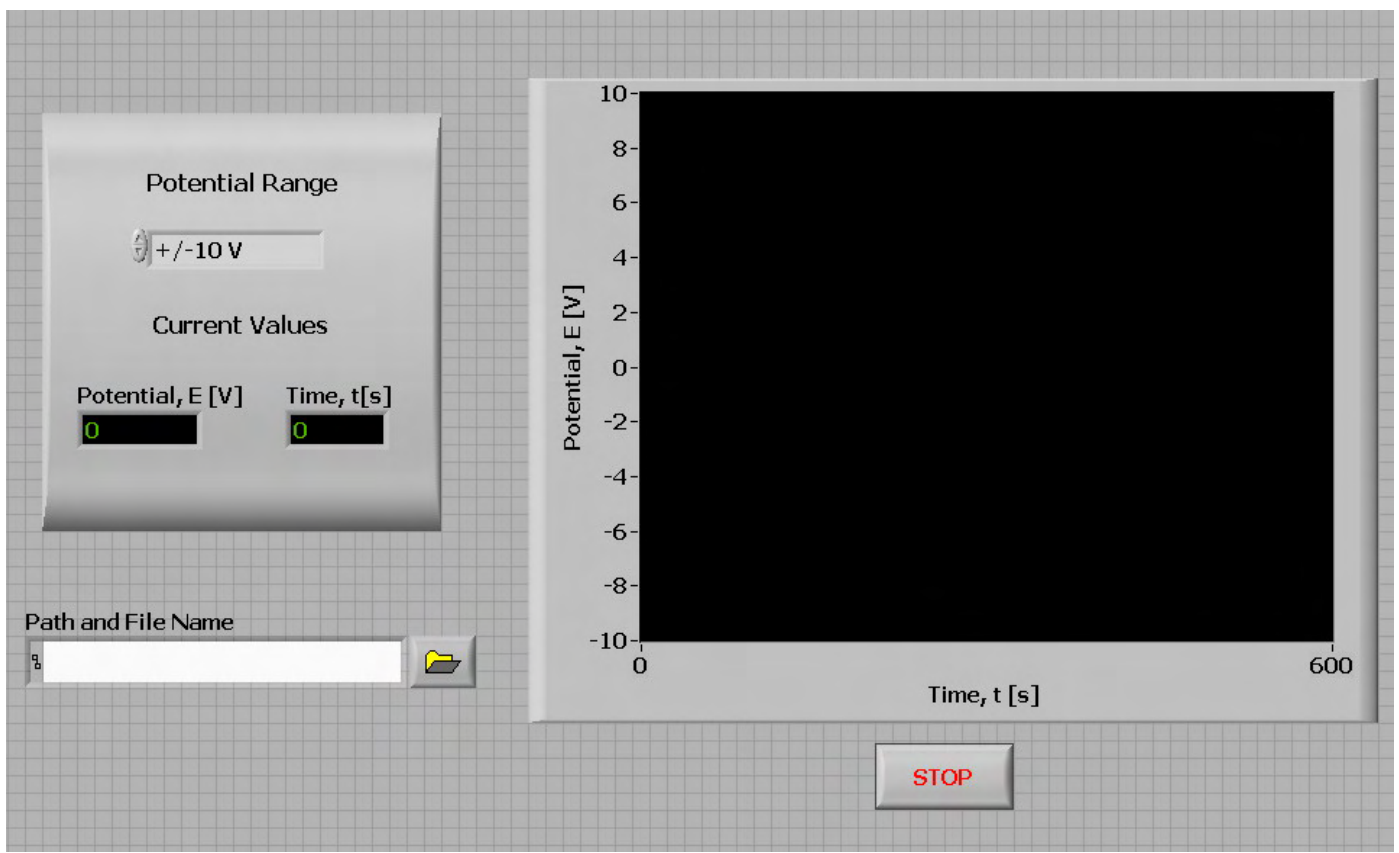

Figure 3. Block diagram of instrument for measuring the open circuit potential.

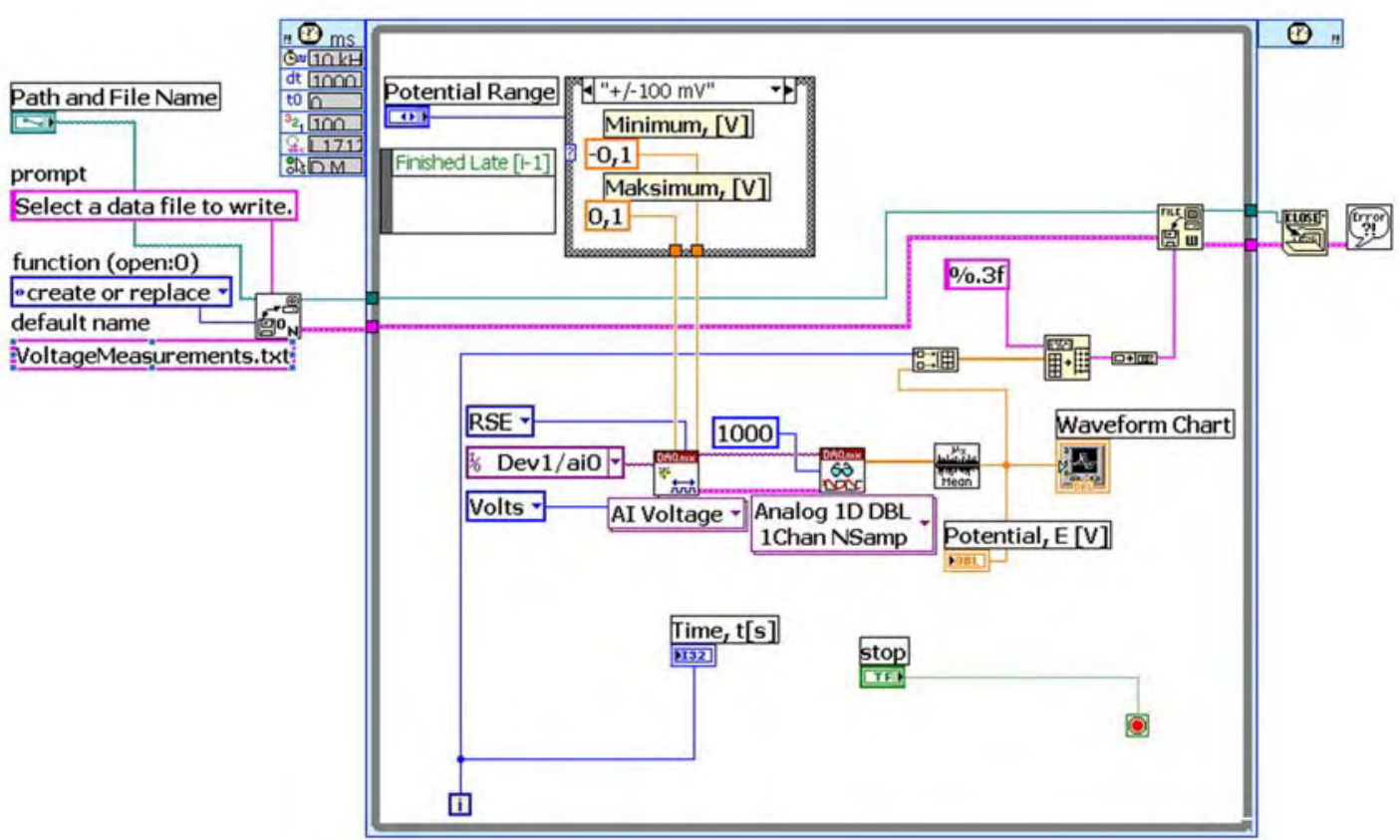

\subsection{Galvanostatic method}

The sequences of initialization as well as the recording into a file are very similar in this method as they are in the previous paragraph, so that they will not be described further. The voltage reading is done on the AICH0 channel and after the process of averaging, correcting and reducing values for the entered value of the open circuit potential $\left(E_{o c}[\mathrm{~V}]\right)$ has been carried out the overvoltage is obtained as a response to the galvanostatic excitation. It has been shown on the indicator eta [V] as well as on the diagram as the time function. 
The current intensity is achieved by the voltage on the block output DAQmax Write in relation to $1 \mathrm{~V} / 10 \mathrm{~mA}$. The voltage to current conversion is solved by the hardware within the scope of the outside interface. The length of the galvanostatic impulse is assigned by controlling $t_{p}[s]$, it is compared with the current time and by IF block it defines the end of the impulse. The measure of the relaxing overvoltage continues until the assigned total time $t_{u}[s]$ has been achieved as it is regulated by comparators and the condition for stopping the time loop (Timed Loop).

In Figure 5 the control panel with the controls for the assigned current $I[\mathrm{~mA}]$, the duration of the impulse $t_{p}[s]$, open circuit potential $E_{o c}[V]$, total time $t_{u}[s]$ as well as indicators of the actual current, overvoltage and time has been shown. On the panel there is a $\mathrm{E}=\mathrm{f}(\mathrm{t})$ graph.

Figure 4. Front panel of the instrument for galvanostatic method.

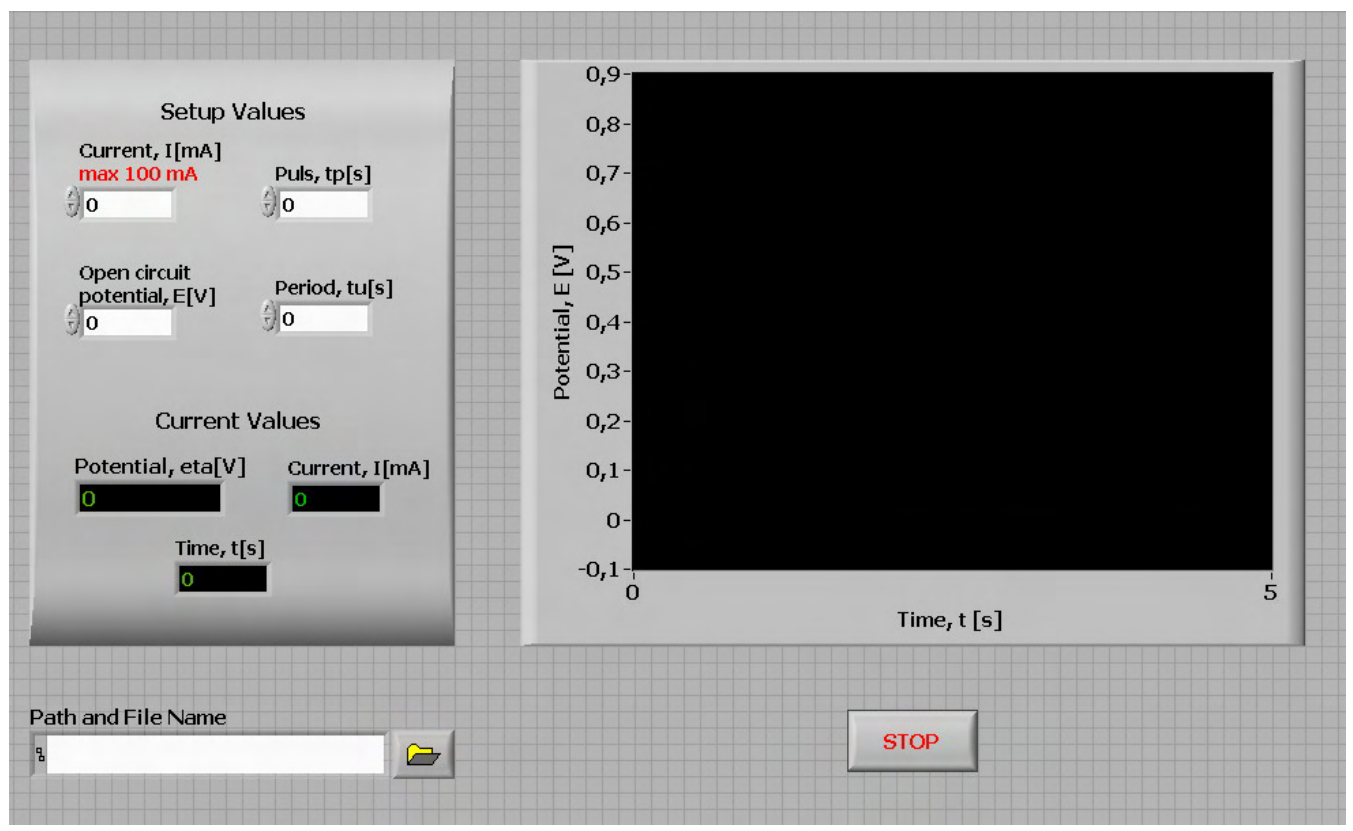

Figure 5. Block diagram of the instrument for galvanostatic method.

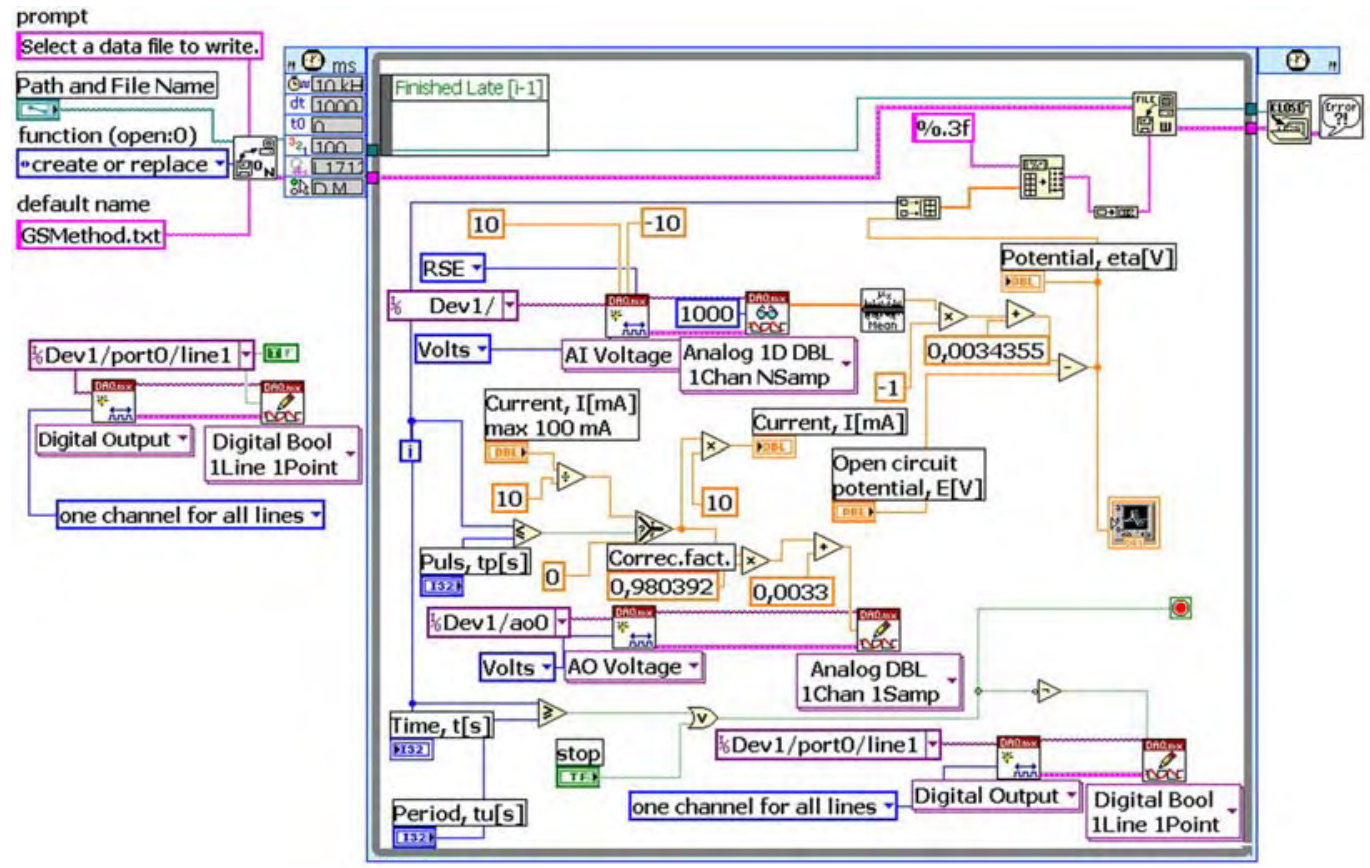




\subsection{Potentiostatic method}

The scheme of application for the potentiostatic method is given in Figure 7. Here, the duration of the pulse is first defined, then $t_{p}$ and IF by the block, and the total time of the experiment by the given $\mathrm{t}_{\mathrm{u}}$ and the condition of stopping the time loop (Timed Loop).

As it is usual with all voltage methods, and similarly here - the current reading is on the first input channel, and the assigned voltage is on the zero output channel.

Figure 6. Front panel of the instruments for potentiostatic method.

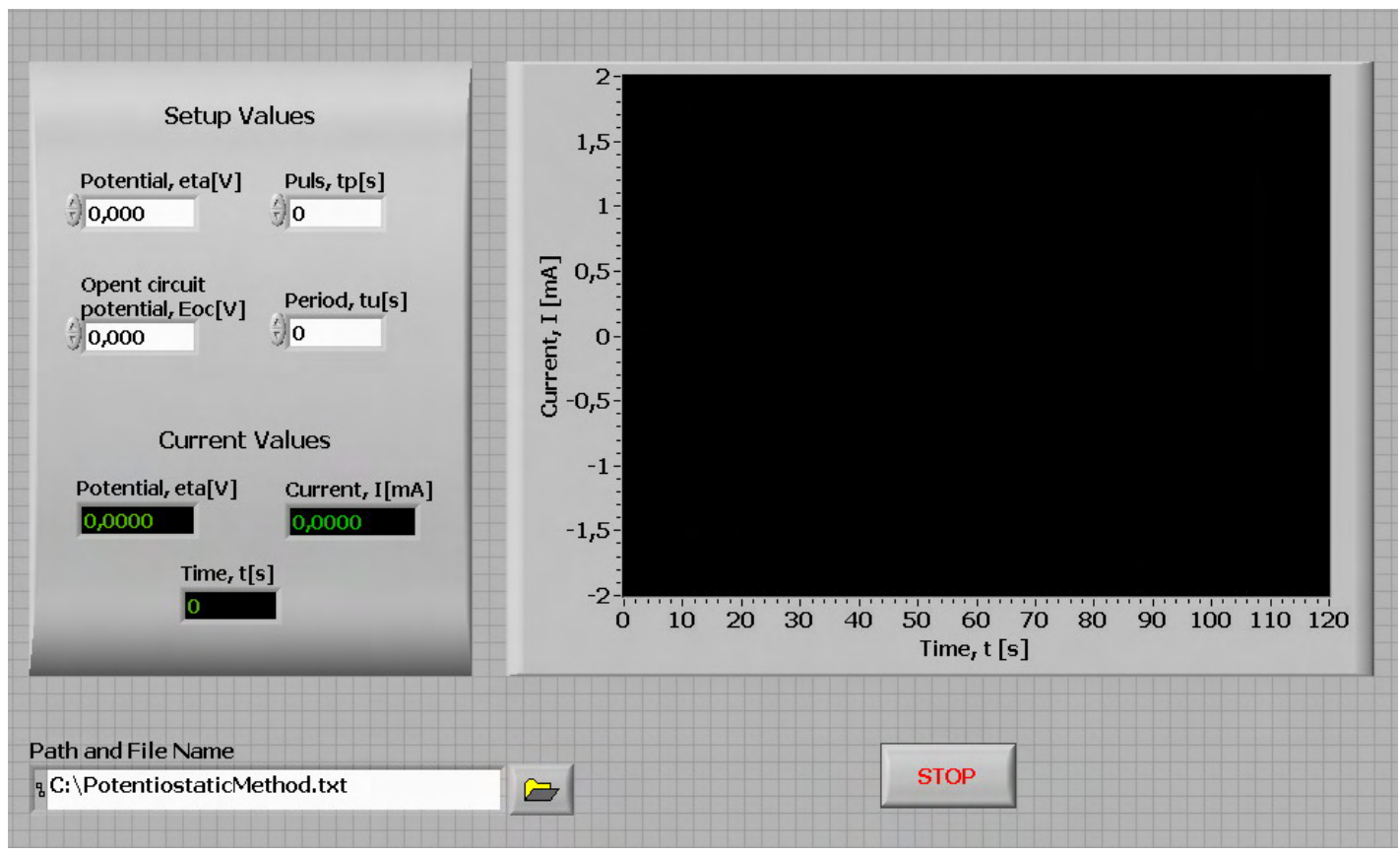

Figure 7. Block diagram of the instruments for potentiostatic method.

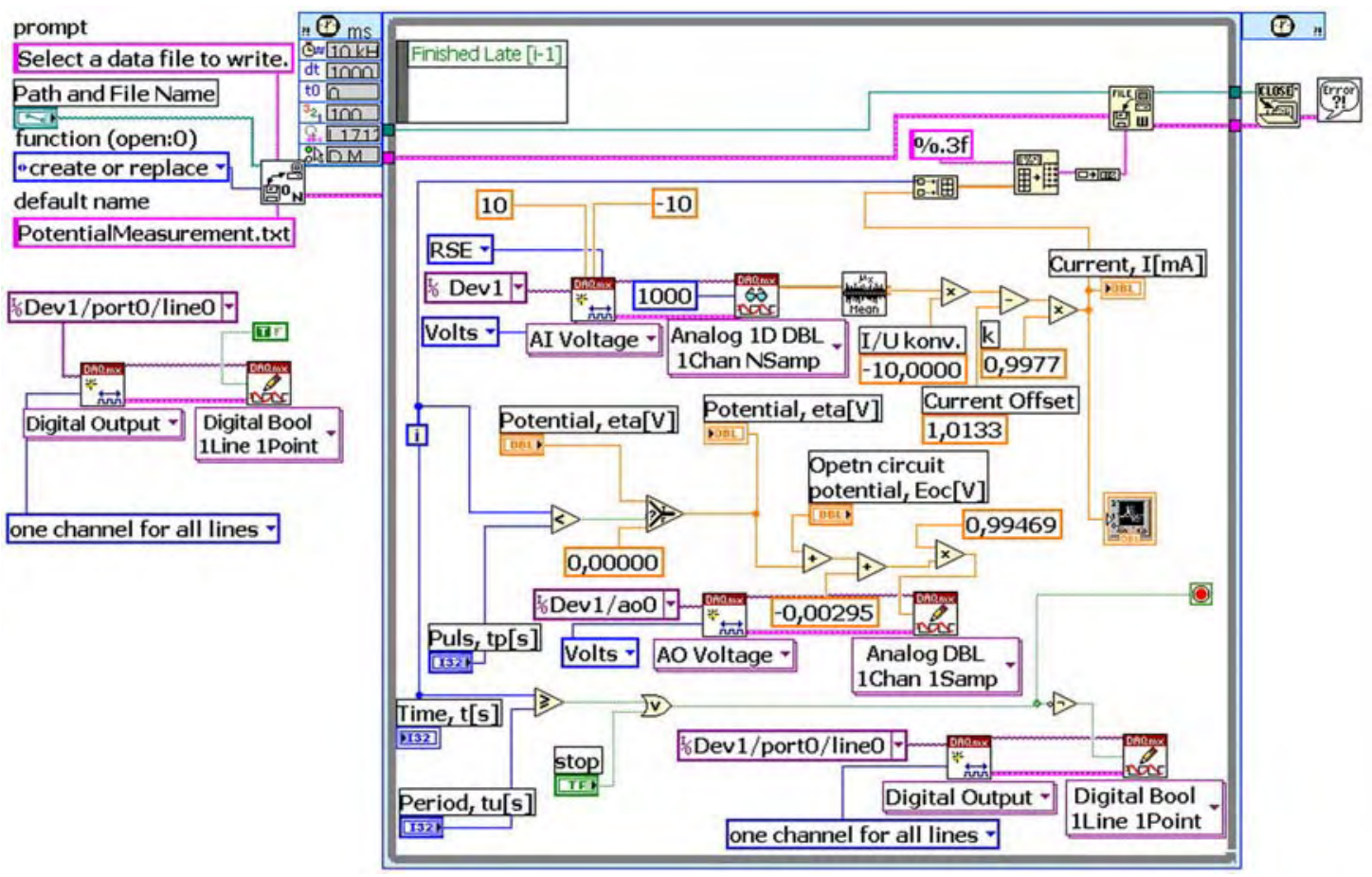




\subsection{Cyclic voltammetry}

In this method, the current measurement (channel AICH1) and assigning of the output voltage (channel AOCH0) are the same with the potentiostatic method, but the current value is calculated in the following way:

$$
\begin{array}{ll}
E=k \cdot t+E_{\text {beg }} & \text { for } t \leq t_{1} \\
E=-k \cdot t+2 E_{\text {fin }}-E_{\text {beg }} & \text { for } t>t_{1}
\end{array}
$$

where: $E$ - is current value of the voltage; $t$ - is time, $k=d E / d t$ - the assigned sweep rate; $t_{1}=\left(E_{\text {fin }}-E_{\text {beg }}\right) / k$ - is the time duration of the voltage rate, $E_{b e g}-$ is the beginning of the voltage; $E_{\text {fin }}$ - is the final value of the voltage.

Functionality of VI for cyclic voltammetry is presented through algorithmic scheme in Figure 8.

Figure 8. Algorithmic scheme of VI for cyclic voltammetry.

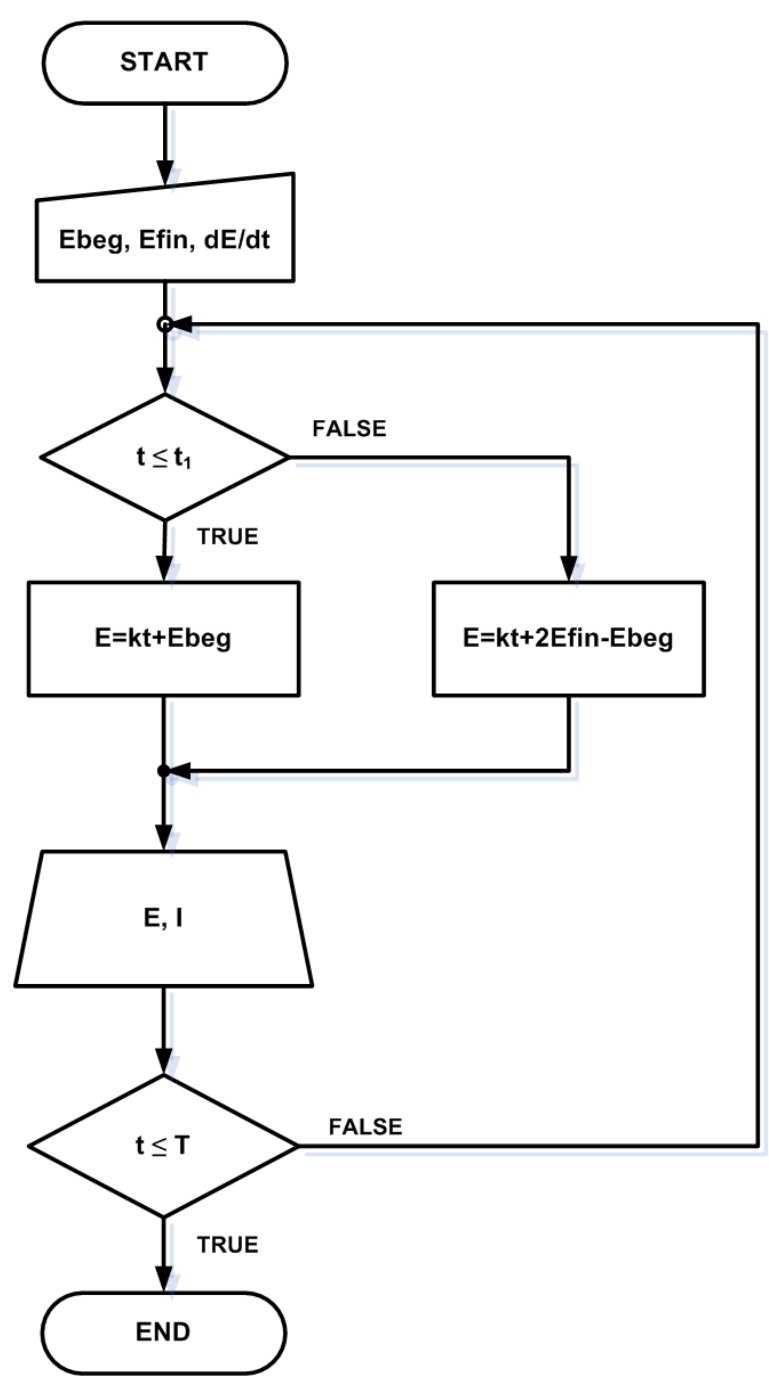

On the instrument front panel (Figure 9), the controls for assigning the beginning and final voltage values, the sweep rates as well as the indicators for current values and graph $\mathrm{i}=\mathrm{f}(\mathrm{E})$ are installed. 
Figure 9. Front panel of the instrument for cyclic voltammetry.

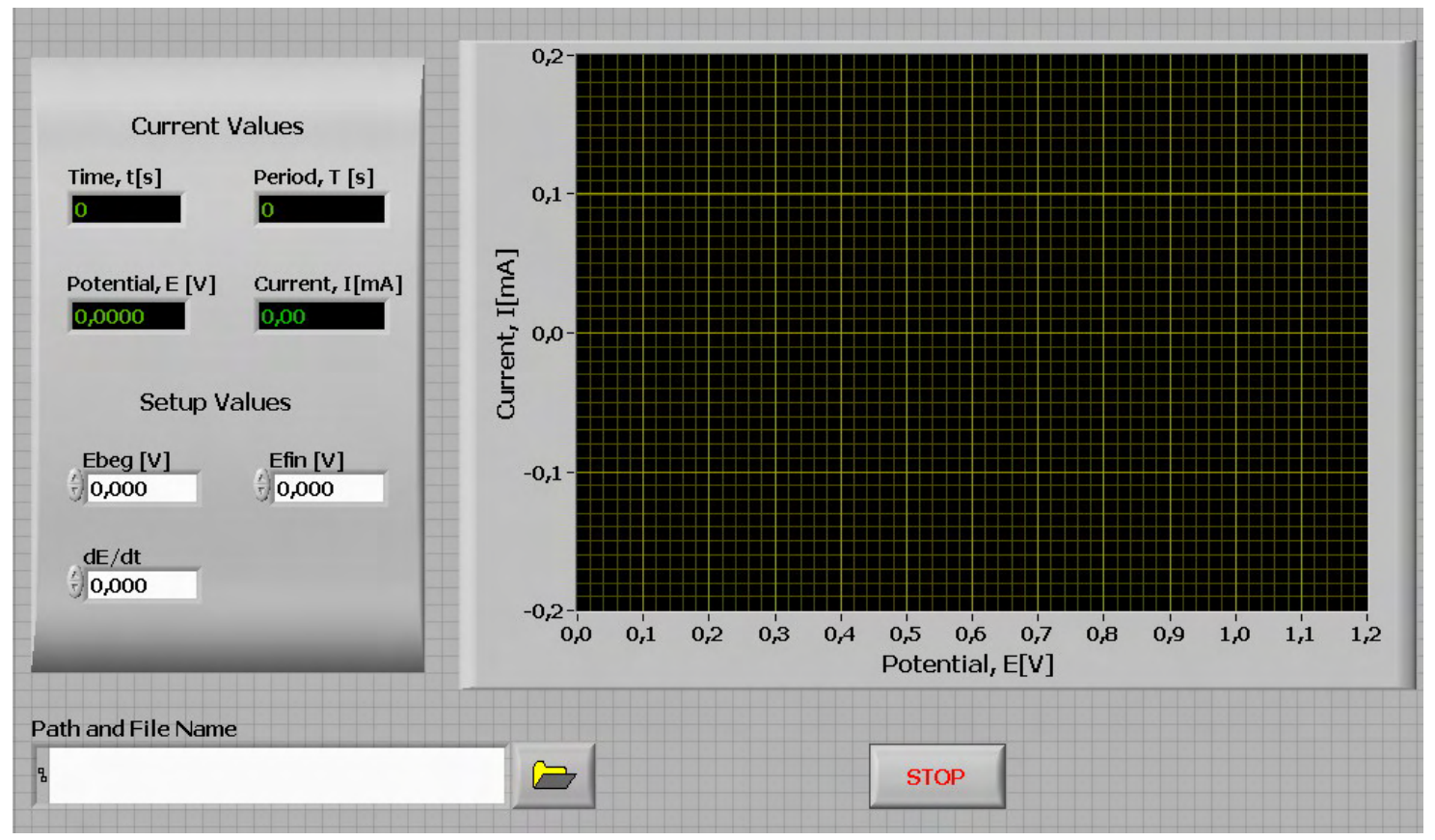

Figure 10. Block diagram of the instrument for cyclic voltammetry.

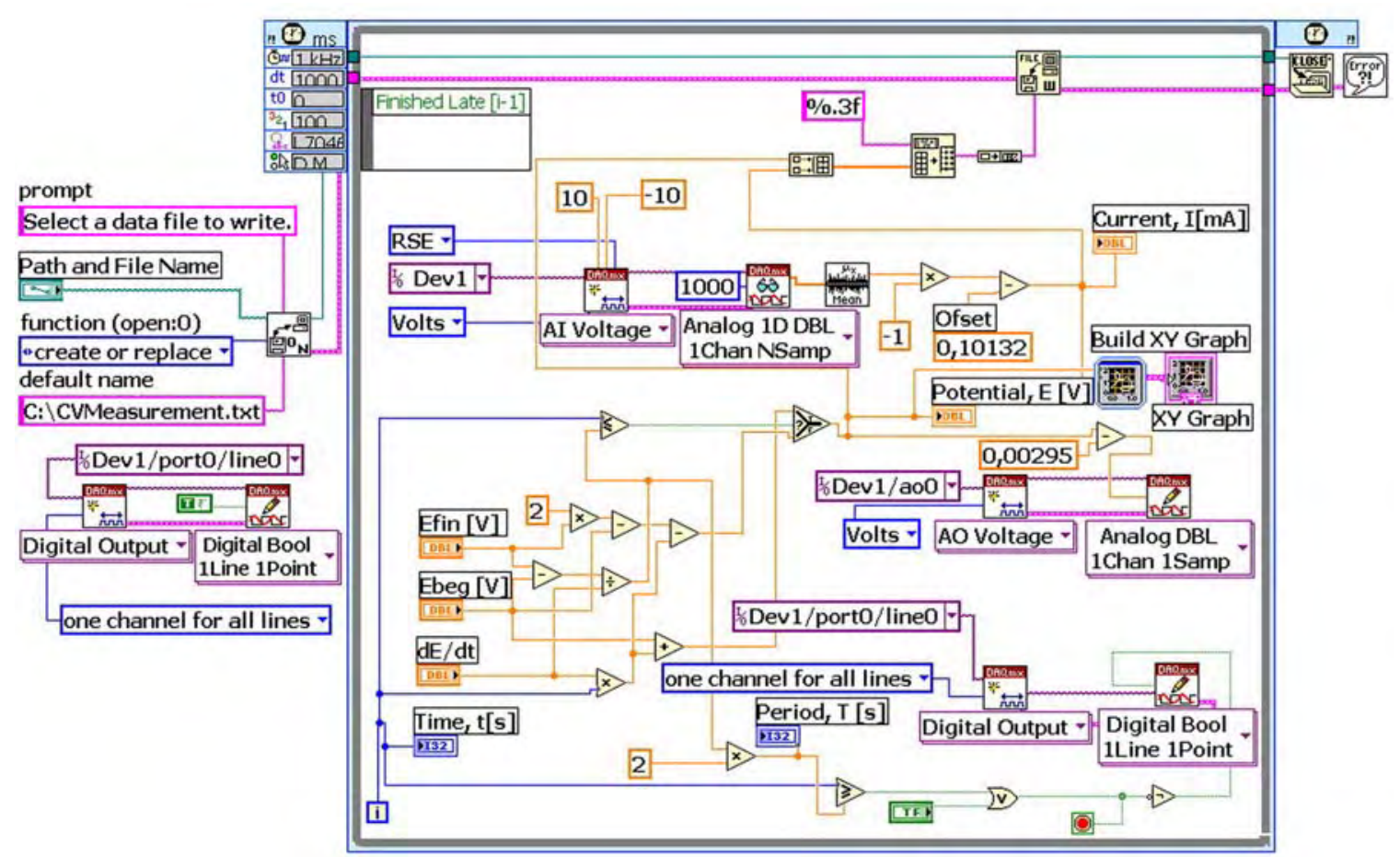

\subsection{Electrochemical impedance of spectroscopy}

This method differs from the previously described in the fact that before any measurement has been done, on the outlet there has been applied a direct (DC) voltage to last long enough in order to achieve a stationary regime, and then the current measurement can be started as a response to a complex excitation (superimposed alternating voltage of an order of magnitude milivolts on DC level up to 10V). 
Installing the DC regime has been solved by a FOR loop which contains a module DAQmx Write, through which is possible to set the direct voltage on the analog output channel (AOCH0).

The assigned direct voltage is $E_{D C}$, and $t_{0}$ is the time of the regime duration. After the DC regime expires, the initial WHILE loop starts, in which the current value of the alternate voltage of the assigned amplitude $\mathrm{E}_{\mathrm{mAC}}$ and the calculated frequencies (block DAQmx Write), the channel (AOCH1) have been generated. The frequency is calculated in the external loop according to octaves, in relation to the assigned values starting from $f_{\text {min }}$ to $f_{\text {max }}$. The lowest frequency can be $1 \mu \mathrm{Hz}$ necessary for systems with high capacitances of containing some high RC couple.

Figure 11. Front panel of the instruments for electrochemical impedance spectroscopy.

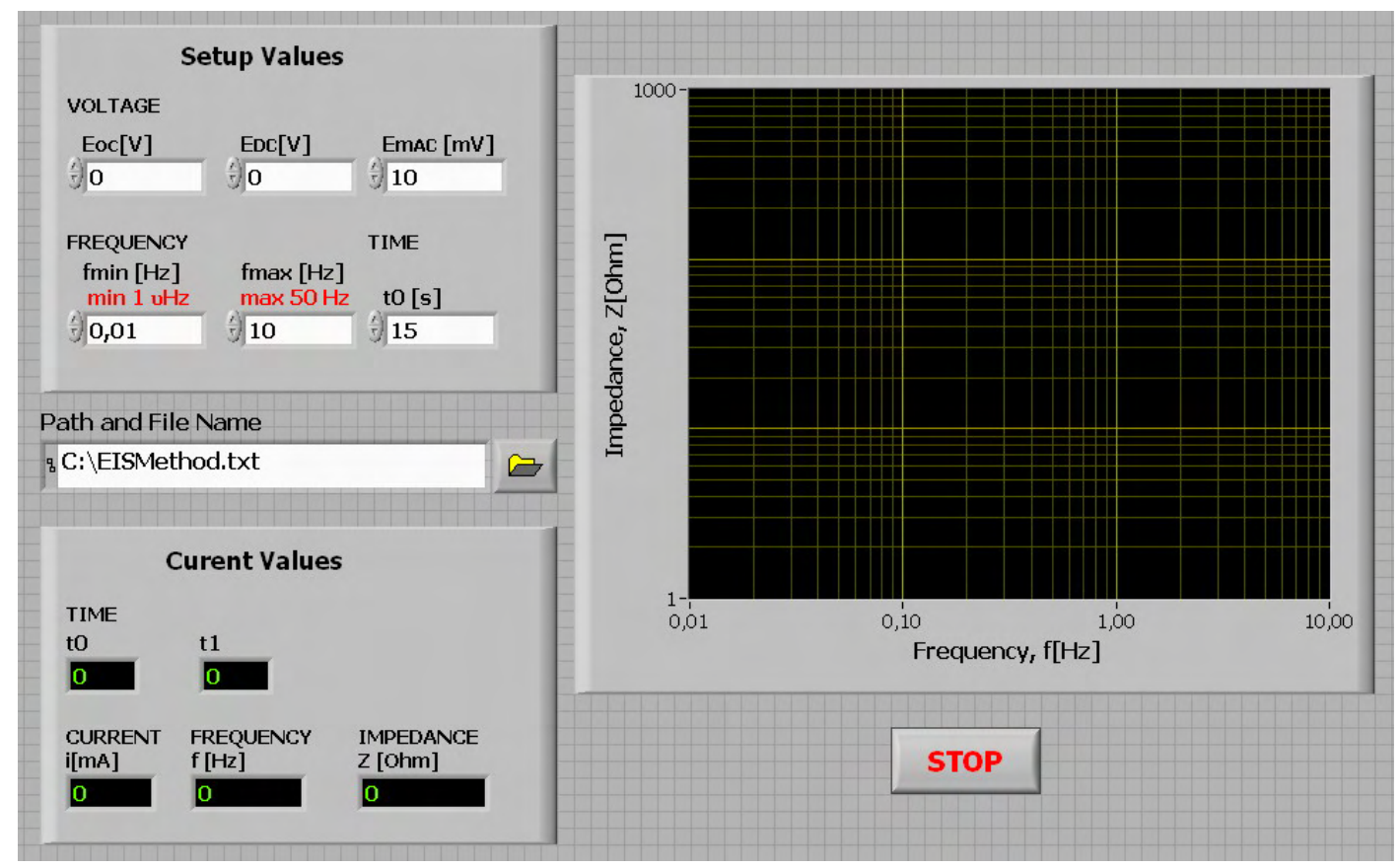

Figure 12. Block diagram of the instruments for electrochemical impedance spectroscopy.

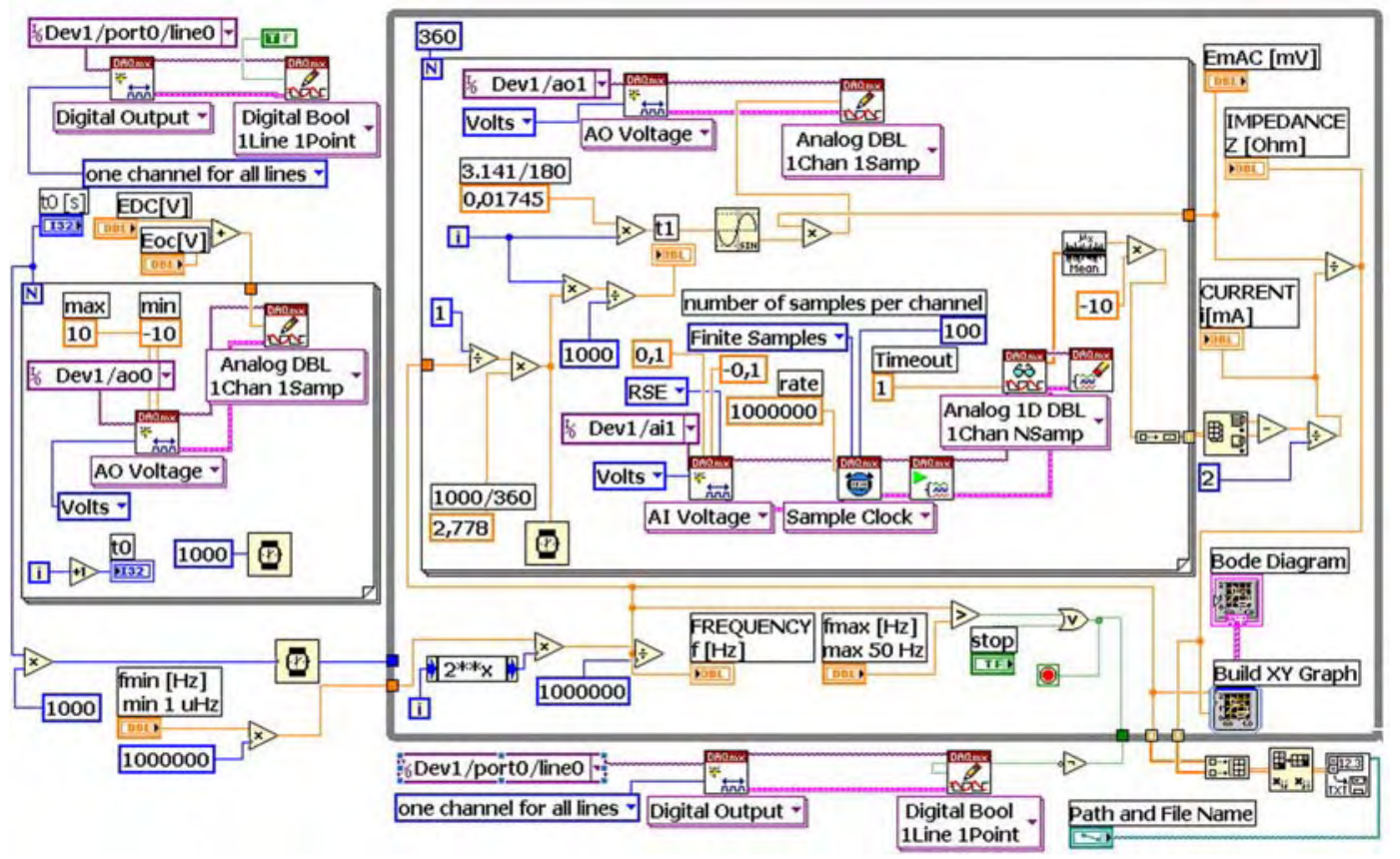


The generated alternating voltage is being superimposed with already installed DC voltage and they both make together the excitation of the electrochemical system.

The current response, which in the pseudo stationary regime has also got the sinusoidal shape with a DC component, is measured on the analog input channel AICH1, being averaged, converted into an array and led in a block Array Max\&Min. On the base of this block it is possible to calculate the average maximal value of the superimposed component of the voltage excitation so that the outcome is the module of system impedance, which is memorized and graphically displayed as a function of the logarithm of the frequency - Bode's diagram of an impedance.

In Figure 11 the front panel of instrument with controllers for assigning the values $E_{D C}, t_{0}, E_{m A C}$, $f_{\min }, f_{\max }$ and and $E_{o c}$, as well as indicators for current values and the diagram $Z=f(f)$ in the logarithm scale are shown.

\section{System Realization and Testing}

A photograph of the realized described system for electrochemical measurements is given in Figure 13. In the figure the galvanic cell is replaced by its physical model that is used for testing the measurement system itself, but the measurement methods, too.

Figure 13. Photograph of the electrochemical measurement system.

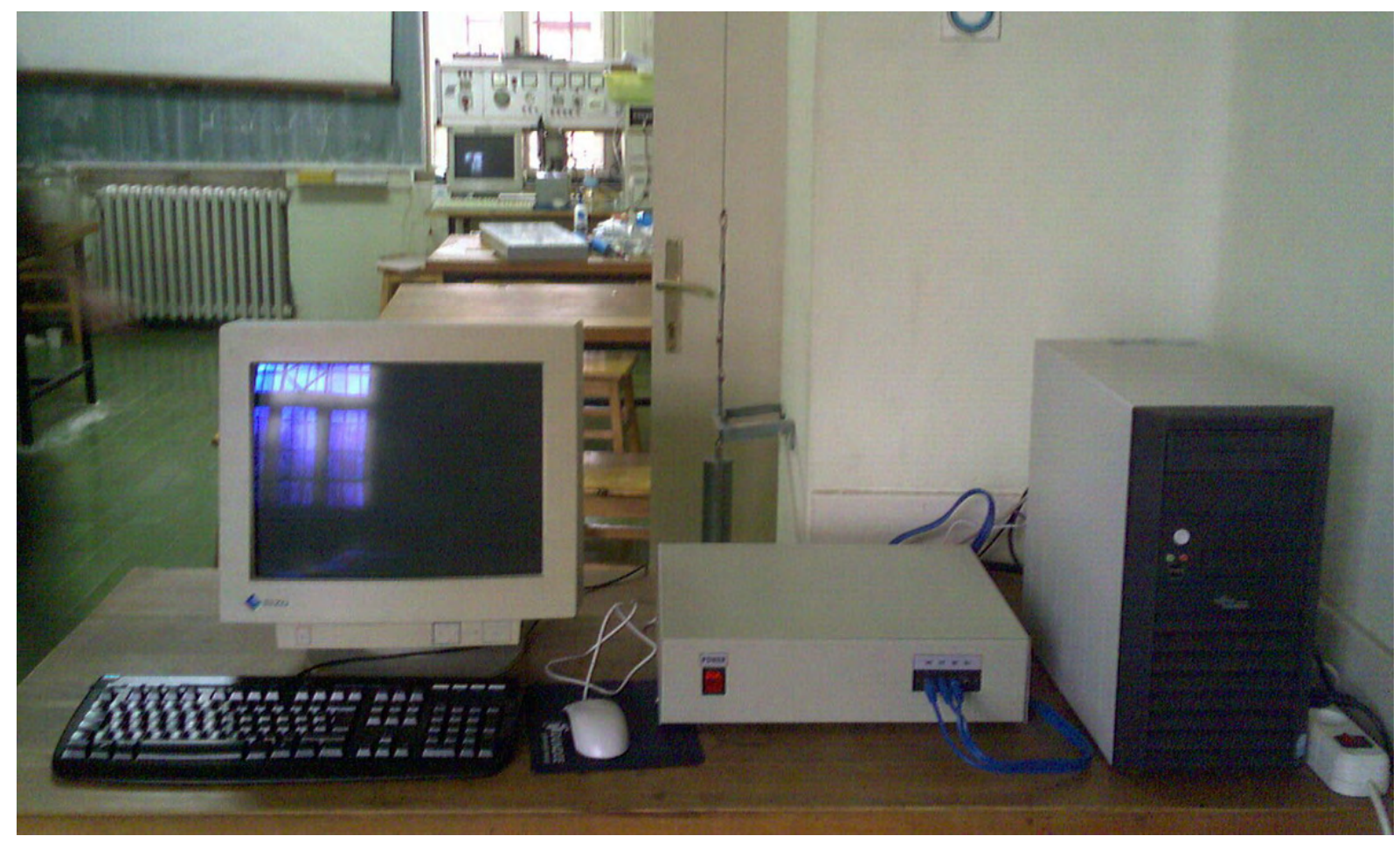

The system is calibrated using high accurate measurement instruments predicted for laboratory instruments adjusting (PRIMA B7-21A, PRIMA B7-38 and PHILIPS 5712). Measurement error less than $0.5 \%$ in all ranges is achieved. The results obtained using this system are compared with those obtained in the same conditions using commercial galvanostat-potentiostat AMEL 551, and also with the results obtained by simulation in ORCAD software package. Excellent agreement of compared data can be seen in Figures 14 and 15. 
Figure 14. Galvanostatic curve obtained on chalcocite in $1 \mathrm{M} \mathrm{H}_{2} \mathrm{SO}_{4}+0,1 \mathrm{M} \mathrm{CuSO}$ using current intensity of $0,5 \mathrm{~mA}$ : PC - result obtained on the system described in the paper, AMEL - results obtained using commercial system.

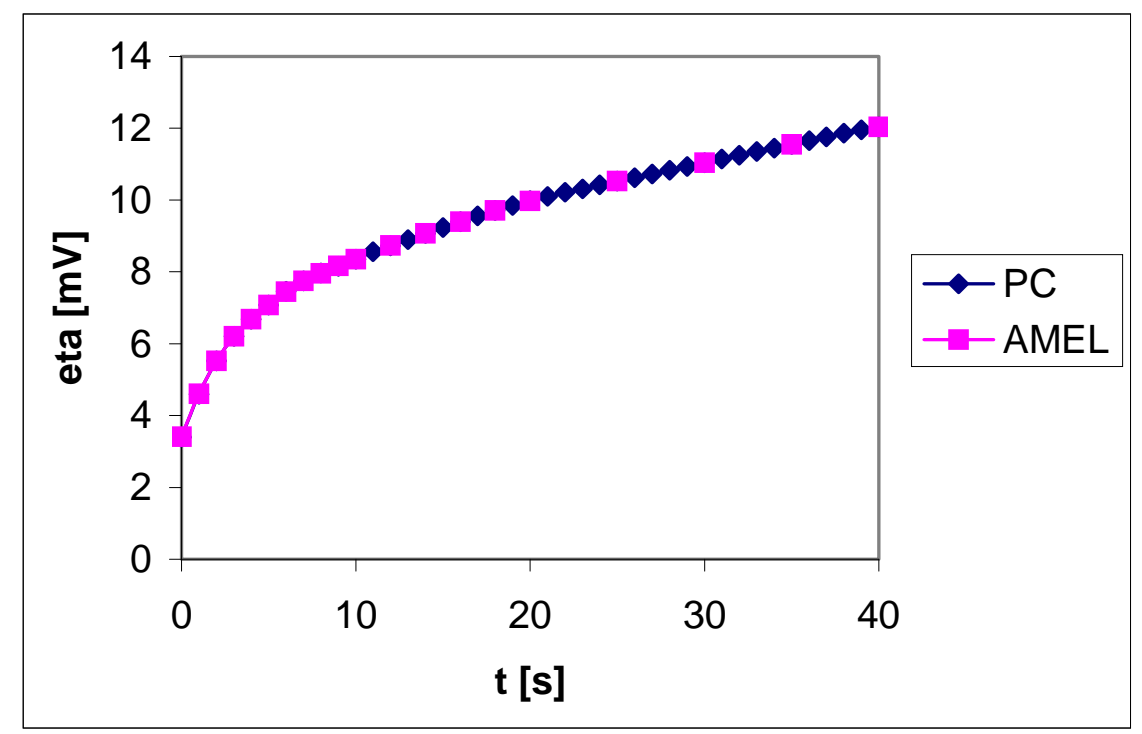

Figure 15. Potentiostatic curve for chalcocite in $1 \mathrm{M} \mathrm{H}_{2} \mathrm{SO}_{4}+0,1 \mathrm{M} \mathrm{CuSO}$ obtained using an excitation voltage of $20 \mathrm{mV}$ : PC - result obtained on the system described in the paper, ORCAD - results obtained by simulation.

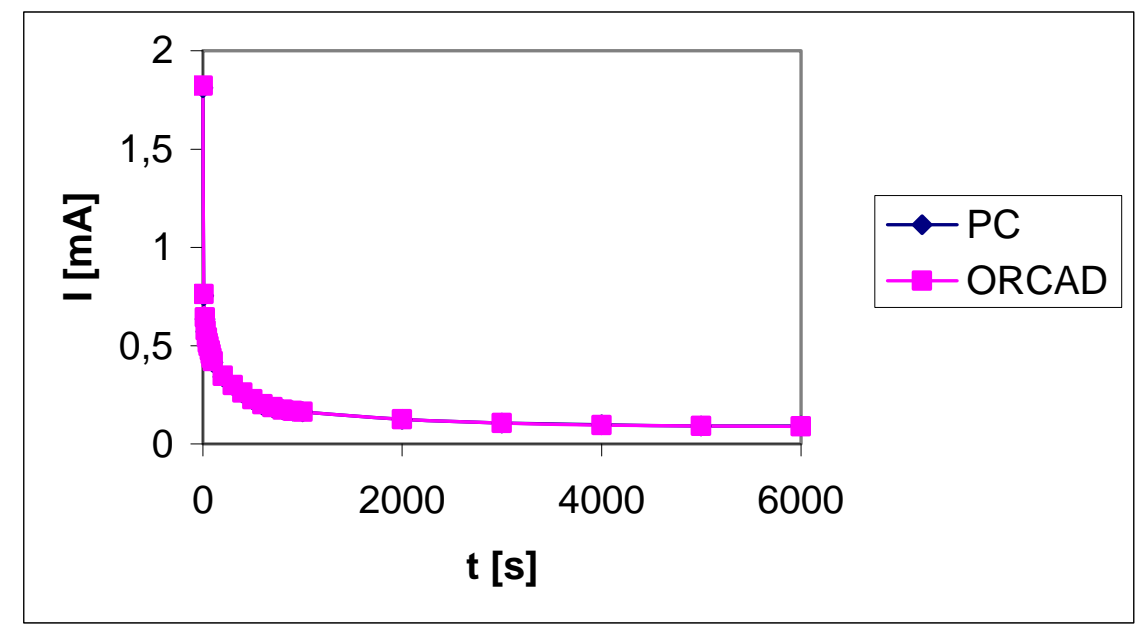

\section{References and Notes}

1. Scholz, F. Electroanalytical methods: guide to experiments and application. Web reference: http://www.chemweb.com/arforms?fid=31.

2. Skryabin, I.L.; Evans, G.; Frost, D.; Vogelman, G.; Bell, J.M. Testing and Control Issues in Large Area Electrochromic Films and Devices. Electrochim. Acta 1999, 44, 3203-3209.

3. Stevic, Z.; Rajcic-Vujasinovic, M. System for Electrochemical Investigations Based on PC and LabVIEW Package. Chem. Ind. 2007, 61, 1-6.

4. Bozeman, R.J. System of Memorizing Maximum Values. US Patent 5539402, 1996. 
5. Rajcic-Vujasinovic, M.; Stankovic, Z.; Stevic, Z. The Consideration of the Electrical Circuit Analogous to the Copper or Coppersulfide/Electrolyte Interfaces Based on the Time Transient Analysis. Russ. Electrochem. 1999, 35, 347- 354.

6. Andrieu, X.; Josset, L. Bifunctional Electrode for an Electrochemical Cell or a Supercapacitor and a Method of Producing it. Patent: Accumulateurs Fixes et de Traction S A SAFT FR, US Patent 5811205, 1998.

7. Park, J.; Mackay, S. Practical Data Acquisition for Instrumentation and Process Control; Newnes: Oxford, 2003.

8. National Instruments. M Series User Manual. November 2006. Part Number: 371022G-01. Web reference: http://www.ni.com.

9. National Instruments. LabVIEW development guidelines. NI Corporation, 2006.

10. Kis, P.; Kuczmann, M.; Fuzi, J.; Ivanyi, A. Hysteresis Measurement in LabVIEW. Physica B 2004, 343, 357-363.

11. Grinstead, B.; Parten, M.E. Biomedical Signal Acquisition Using “LabVIEW”. Proc. $11^{\text {th }}$ IEEE Symp. Comput.-Based Med. Syst. CBMS 1998; pp. 157-161.

(C) 2008 by MDPI (http://www.mdpi.org). Reproduction is permitted for noncommercial purposes. 